\title{
Lower Normal Topological Spaces and Lower Continuity
}

\author{
Milan R. TASKOVIĆ
}

\begin{abstract}
In this paper we formulate a new structure of topological spaces which we call it lower normal spaces. This concept of spaces is directly and nature connection with the lower transversal continuous mappings on topological spaces. In this sense, we shall study spaces in which it is possible in the same way to separate two disjoint closed sets by a lower continuous real valued function. Applications in nonlinear functional analysis are considered. The concept of lower normal spaces is closely connected with the concept of normal topological spaces and the results of Alexandroff, Urysohn, Tietze, Lebesgue, Dieudonné, Tychonoff, Lefschetz, and Vietoris.
\end{abstract}

\section{Introduction And Lower Continuity}

If we agree that the mathematical notion of neighborhood corresponds to the intuitive idea of "some proximity", then we can express the following new definition of a new continuity in the following sense.

Let $X$ and $Y$ be topological spaces, $f$ a mapping of $X$ into $Y, O(x)$ is the set of all neighborhood of $x \in X$, and let $O(y)$ the set of all neighborhood of $y \in Y$. We say that $f$ is lower continuous at $x_{0} \in X$ iff for every neighborhood $V$ of $f\left(x_{0}\right)$ in $Y$, there exists a neighborhood $U$ of $x_{0}$ in $X$ such that $V \subset f(U)$. Also, $f$ is said to be lower continuous in $A \subset X$ if it is lower continuous at every point $x \in A$.

This means that $f$ is lower continuous at $x_{0} \in X$ iff for every neighborhood $V \in O\left(f\left(x_{0}\right)\right)$ there exists a neighborhood $U \in O\left(x_{0}\right)$ such that $V \subset f(U)$.

For example, let $\left(X, d_{X}\right)$ and $\left(Y, d_{Y}\right)$ be metric spaces, $f$ mapping of $X$ into $Y$, and $x_{0} \in X$. From the preceding definition, to say that $f$ is lower continuous at $x_{0}$ means that: for every $\varepsilon>0$, there exists $\delta>0$ such that for every $x \in X$ we have that

$$
d_{X}\left(x, x_{0}\right)>\delta \text { implies } \quad d_{Y}\left(f(x), f\left(x_{0}\right)\right)>\varepsilon .
$$

2000 Mathematics Subject Classification. Primary: 54E15; Secondary: 54E35.

Key words and phrases. Topological Spaces, Lower Normal Spaces, Extension of One Lower Continuous Real-Valued Function, Lower Continuous Partitions of Unity. 
A natural illustration for this is on the following transversal lower spaces. In this sense, let $X$ be a nonempty set.

The possibility of defining such notions as limit and continuity in an arbitrary set is an idea which undoubtedly was first put forward by M. Fréchet in 1904, and developed by him in his famous thesis in 1906.

The simplest and most fruitful method which be proposed for such definitions was the introduction of the notion of distance.

In connection with this, first, in Tasković [5] we introduced the concept of transversal (upper and lower) spaces as a natural extension of Fréchet's Kurepa's and Menger's spaces.

Let $X$ be a nonempty set. The function $\rho: X \times X \rightarrow \mathbb{R}_{+}^{0}:=[0,+\infty)$ is called an upper transverse on $X$ (or upper transversal) iff: $\rho[x, y]=\rho[y, x]$, $\rho[x, y]=0$ if and only if $x=y$, and if there is function $\psi:\left(\mathbb{R}_{+}^{0}\right)^{2} \rightarrow \mathbb{R}_{+}^{0}$ such that

$$
\rho[x, y] \leq \max \{\rho[x, z], \rho[z, y], \psi(\rho[x, z], \rho[z, y])\}
$$

for all $x, y, z \in X$. An upper transversal space is a set $X$ together with a given upper transverse on $X$. The function $\psi$ in (As) is called upper bisection function.

On the other hand, the function $\rho: X \times X \rightarrow[0,+\infty]:=\mathbb{R}_{+}^{0} \cup\{+\infty\}$ is called a lower transverse on $X$ (or lower transversal) iff: $\rho[x, y]=\rho[y, x]$, $\rho[x, y]=+\infty$ if and only if $x=y$, and if there is a lower bisection function $d:[0,+\infty]^{2} \rightarrow[0,+\infty]$ such that

$$
\rho[x, y] \geq \min \{\rho[x, z], \rho[z, y], d(\rho[x, z], \rho[z, y])\}
$$

for all $x, y, z \in X$. A lower transversal space is a set $X$ together with a given lower transverse on $X$. The function $d$ in $(\mathrm{Am})$ is called lower bisection function.

Let $(X, \rho)$ be a lower transversal space and $T: X \rightarrow X$. We shall introduce the concept of DS-convergence in a space $X$; i.e., a lower transversal space $X$ satisfies the condition of DS-convergence (or $X$ is DS-complete) iff: $\left\{x_{n}\right\}_{n \in \mathbb{N}}$ is an arbitrary sequence in $X$ and $\sum_{i=1}^{\infty} \rho\left[x_{i}, x_{i+1}\right]=+\infty$ implies that $\left\{x_{n}\right\}_{n \in \mathbb{N}}$ has a convergent subsequence in $X$.

In connection with this, a lower transversal space $X$ satisfies the condition of orbitally DS-convergence (or $X$ is orbitally DS-complete) iff: $\left\{T^{n} x\right\}_{n \in \mathbb{N} \cup\{0\}}$ for $x \in X$ is an arbitrary iteration sequence in $X$ and

$$
\sum_{n=0}^{\infty} \rho\left[T^{n} x, T^{n+1} x\right]=+\infty \quad(\text { for } x \in X)
$$

implies that $\left\{T^{n} x\right\}_{n \in \mathbb{N} \cup\{0\}}$ has a convergent subsequence in $X$.

We notice that in [6] Tasković proved the following statement for a class of expansion mappings. Namely, if $(X, \rho)$ is an orbitally $D S$-complete lower 
transversal space, if $T: X \rightarrow X$, and if there exists a number $q>1$ such that

$$
\rho(T(x), T(y)) \geq q \rho(x, y)
$$

for each $x, y \in X$, then $T$ has a unique fixed point in the lower transversal space $X$.

Let $\left(X, \rho_{X}\right)$ and $\left(Y, \rho_{Y}\right)$ be two lower transversal spaces and let $T: X \rightarrow$ $Y$. We notice, from Tasković [6], that $T$ be lower transversal continuous (or lower continuous) at $x_{0} \in X$ iff for every $\varepsilon>0$ there exists a $\delta>0$ such that the relation

$$
\rho_{X}\left[x, x_{0}\right]>\delta \quad \text { implies } \rho_{Y}\left[T(x), T\left(x_{0}\right)\right]>\varepsilon .
$$

A typical first example of a lower transversal continuous mapping is the mapping $T: X \rightarrow X$ with property (1). Also, the lower transverse $\rho$ need not be lower transversal continuous; but, for an arbitrary metric function $r(x, y)$ the lower transverse of the form $\rho[x, y]:=1 / r(x, y)$ is a lower transversal continuous function. For further facts on the lower transversal continuous mappings see: Tasković [6].

In this sense, for any nonempty set $S$ in the lower transversal space $X$ the diameter of $S$ is defined by

$$
\operatorname{diam}(S):=\inf \{\rho[x, y]: x, y \in S\}
$$

it is a positive real number or $+\infty$, and $A \subset B \operatorname{implies} \operatorname{diam}(B) \leq \operatorname{diam}(A)$. The relation $\operatorname{diam}(S)=0$ holds if and only if $S$ is a one point set. Also, for a point $x_{0} \in X$ we have

$$
\rho\left(x_{0}, S\right):=\sup \left\{\rho\left[x_{0}, s\right]: s \in S\right\} .
$$

Elements of a lower transversal space will usually be called points. Given a lower transversal space $(X, \rho)$, with the bisection funkction $d$ and a point $z \in X$, the open ball of center $z$ and radius $r>0$ is the set

$$
d(B(z, r)):=\{x \in X: \rho[z, x]>r\}
$$

In this sense, we have the following form of convergence on the lower transversal spaces. The convergence $x_{n} \rightarrow x$ as $n \rightarrow \infty$ in the lower transversal space $(X, \rho)$ means that

$$
\rho\left[x_{n}, x\right] \rightarrow+\infty \quad \text { as } \quad n \rightarrow \infty,
$$

or equivalently, for every $\varepsilon>0$ there exists an integer $n_{0}$ such that the relation $n \geq n_{0}$ implies $\rho\left[x_{n}, x\right]>\varepsilon$.

The sequence $\left\{x_{n}\right\}_{n \in \mathbb{N}}$ in the lower transversal space $(X, \rho)$ is called transversal sequence (or lower Cauchy sequence) iff for every $\varepsilon>0$ there is an $n_{0}=n_{0}(\varepsilon)$ such that

$$
\rho\left[x_{n}, x_{m}\right]>\varepsilon \quad \text { for all } n, m \geq n_{0} .
$$


Let $(X, \rho)$ be a lower transversal space and $T: X \rightarrow X$. We notice, from Tasković [6], that a sequence of iterates $\left\{T^{n}(x)\right\}_{n \in \mathbb{N}}$ in $X$ is said to be transversal sequence if and only if

$$
\lim _{n \rightarrow \infty}\left(\operatorname{diam}\left\{T^{k}(x): k \geq n\right\}\right)=+\infty .
$$

In this sense, a lower transversal space is called lower complete iff every transversal sequence converges.

Also, a space $(X, \rho)$ is said to be lower orbitally complete (or lower $T$-orbitally complete) iff every transversal sequence which is contained in the orbit $\mathcal{O}(x):=\left\{x, T x, T^{2} x, \ldots\right\}$ for some $x \in X$ converges in $X$.

We will, in further, denote by $\mathcal{D}([0,+\infty])$ the set of all lower bisection functions $d:[0,+\infty]^{2} \rightarrow[0,+\infty]$ which are increasing satisfying $d(t, t) \geq t$ for every $t \in[0,+\infty]$.

Proposition 1. Let $X, Y, Z$ be topological spaces, $f: X \rightarrow Y, g: Y \rightarrow Z$, $x_{0} \in X$, and $y_{0}=f\left(x_{0}\right) \in Y$. If $f$ is lower continuous at $x_{0}$, and $g$ is lower continuous at $y_{0}$, then $h=g \circ f: X \rightarrow Z$ is lower continuous at $x_{0}$.

Proof. Let $W$ be a neighborhood of a point $z_{0}=h\left(x_{0}\right)=g \circ f\left(x_{0}\right)=g\left(y_{0}\right)$. There exists a neighborhood $V$ of $y_{0}$ such that $W \subset g(V)$, because $g$ is lower continuous at $y_{0}$. Since $f$ is lower continuous at $x_{0}$, thus there exists a neighborhood $U$ of $x_{0}$ such that $V \subset f(U)$. Therefore, $W \subset g(V) \subset$ $g(f(U))=h(U)$, whence the statement.

Corollary 1. Let $X, Y, Z$, be topological spaces and $f: X \rightarrow Y, g: Y \rightarrow Z$ be lower continuous mappings. Then $h=g \circ f: X \rightarrow Z$ is a lower continuous mapping.

If $f: X \rightarrow Y$ is a lower continuous mapping and $A \subset X$, then the restriction $g=f \mid A: A \rightarrow Y$ is a lower continuous mapping. In this sense, $f$ is called lower continuous extension of $g$.

Proposition 2. Let $X, Y$ be topological spaces and $f$ a bijective mapping of $X$ into $Y$. The following properties are equivalent:

(a) $f$ is lower continuous;

(b) for every open set $V \subset Y, f^{-1}(V)$ is a closed set in $X$;

(c) for every closed set $F \subset Y, f^{-1}(F)$ is an open set in $X$;

(d) for every set $A \subset X$ is $f(\operatorname{Int} A) \subset \mathrm{Cl} f(A)$, i.e., Int $A \subset f^{-1}(\mathrm{Cl} f(A))=\operatorname{Int} f^{-1}(\mathrm{Cl} f(A))$.

Proof. (a) implies (b). Let $V$ be an open set in $Y$. For every point $x \in$ $f^{-1}(V), V$ is a neighborhood of point $f(x)$, thus there exists a neighborhood $U$ of the point $x$ such that $V \subset f(U)$, i.e., $x \in U \supset f^{-1}(V)$. This means that $x$ is an exterior point of $f^{-1}(V)$. Hence $f^{-1}(V) \supset \mathrm{Cl} f^{-1}(V)$, i.e., $f^{-1}(V)$ is a closed set in $X$. 
(b) implies (c). Let $F \subset Y$ be a closed set. Then $V=Y \backslash F$ is an open set. From (b) we obtain that $f^{-1}(Y \backslash F)$ is closed in $X$. Since $f^{-1}(F)=$ $X \backslash f^{-1}(Y \backslash F)$ we have that $f^{-1}(F)$ is open in $X$.

(c) implies (d). Let $A$ be an arbitrary subset of $X$. Hence, from (c), $f^{-1}(\mathrm{Cl} f(A))$ is an open set in $X$ such that

$$
A=f^{-1}(f(A)) \subset f^{-1}(\mathrm{Cl} f(A))=\operatorname{Int} f^{-1}(\mathrm{Cl}(f(A))) ;
$$

and thus $\operatorname{Int} A \subset f^{-1}(\mathrm{Cl} f(A))$, i.e., $f(\operatorname{Int} A) \subset \operatorname{Cl} f(A)$.

(d) implies (a). Let $A$ be an arbitrary set in $X$. Then, from (d), for $V=$ $\mathrm{Cl} f(A)$ and $U=\operatorname{Int} f^{-1}(\mathrm{Cl} f(A))$ we obtain $U=f^{-1}(V)$ as a neighborhood of $x$ for which we have $V=f(U)$.

The proof is complete.

\section{Lower Normal Spaces}

We shall now study spaces in which it is possible in the same way to separate two disjunct closed sets by a lower continuous real valued function.

A topological space $X$ is said to be lower normal if it is Hausdorff and satisfies the following fact: If $A$ and $B$ are any two disjoint closed subsets of $X$, there exists a bijection lower continuous mapping of $X$ into $[0,1]$ which is equal to 0 at every point of $A$ and to 1 at every point of $B$.

We notice that this notation very connection with the notation of normal topological spaces. The class of normal spaces was defined by Tietze [8] and by Alexandroff and Urysohn [10]. But, the normality property appeared earlier in Vietoris [11]. In connection with this, Urysohn's lemma was established by Urysohn [10]; but the fact that every second countable regular space is normal was proved by Tychonoff [9].

Theorem 1. A topological space $X$ is lower normal if and only if $A$ and $B$ are any two disjoint closed subsets of $X$ such that then there exist two disjoint open sets $U, V$ satisfying $U \subset A$ and $V \subset B$.

Before of a proof of this statement I present the following characterization lower normality of topological spaces. It is the following result.

Proposition 3. A topological space $X$ is lower normal if and only if for given any closed set $A$ and any open neighborhood $V$ of $A$, there exists an open neighborhood $W$ of $A$ such that $V \subset \mathrm{Cl} W$.

Proof. If there is a lower continuous mapping $f: X \rightarrow[0,1]$ which is equal to 0 on $A$ and to 1 on $B$, and if we put $U(t):=f^{-1}([0, t])$ for each $t \in[0,1]$, then we obtain defined a family of open sets in $X$, indexed by $[0,1]$, such that: (a) $U(0) \subset A$, (b) $C U(1) \subset B$ where $C$ denoted complement, and (c) for each pair of real numbers $0 \leq t<t^{\prime} \leq 1$ we have $U(t) \subset \mathrm{Cl} U\left(t^{\prime}\right)$ for $U(t)$ which included as a subset and the open set of $f^{-1}\left(\left[0, t^{\prime}\right]\right)$.

Conversely, suppose that we have defined a family $(U(t))$ of open sets, for $0 \leq t \leq 1$, with these three properties (a), (b), and (c). For each $x \in X$, 
put $g(x)=1$ if $x \in C U(1)$, and if $x \in U(1)$ let

$$
g(x)=\inf \{t \in[0,1]: x \in U(t)\} .
$$

Thus $0 \leq g(x) \leq 1$ for each $x \in X, g(x)=0$ on $A, g(x)=1$ on $B$. Also, $g$ is lower continuous on $X$, for if we put $g(x)=r$, we have $|g(y)-g(x)|^{-1} \geq \varepsilon$ for all $y \in U(r+\varepsilon) \cap C(\mathrm{Cl} U(r-\varepsilon))$, which is a neighborhood of $x$ by $U\left(t^{\prime}\right) \subseteq \mathrm{Cl} U(t)$. In this case we have conventions that $U(r+\varepsilon)=X$ if $r+\varepsilon>1$, and $U(r-\varepsilon)=\emptyset$ if $r-\varepsilon<0$.

The proof is completed.

Proof of Theorem 1. If $X$ is a lower normal topological space and if $f$ is a lower continuous mapping of $X$ into $[0,1]$ which is equal to 0 on $A$ and to 1 on $B$, then the open sets $f^{-1}(\{0\})$ and $f^{-1}(\{1\})$ are subsets of $A$ and $B$ respectively and do not intersect.

For proof of the converse statement will be proved if we can define a family $(U(t))$ of open sets satisfying conditions (a), (b) and (c) above in Proposition 3; to do this we use of Proposition 3.

Take $U(1) \subset C B$; since $U(1) \subset A$ there exists an open set $U(0)$ such that $U(0) \subset A$ and $U(1) \subset U(0)$ by (b). Suppose then that for each dyadic number $k / 2^{n}\left(k=0,1, \ldots, 2^{n}\right)$ we have defined open set $U\left(k / 2^{n}\right)$, these sets being such that $U\left((k+1) / 2^{n}\right) \subset \mathrm{Cl} U\left(k / 2^{n}\right)$ for $0 \leq k \leq 2^{n}-1$. For each dyadic number $(2 k+1) / 2^{n+1}\left(0 \leq k \leq 2^{n}-1\right)$ there exists by Proposition 3 an open set $U\left((2 k+1) / 2^{n+1}\right)$ such that

$$
U\left((2 k+1) / 2^{n+1}\right) \subset \mathrm{Cl} U\left(k / 2^{n}\right)
$$

and

$$
U\left((k+1) / 2^{n}\right) \subset \mathrm{Cl} U\left((2 k+1) / 2^{n+1}\right),
$$

hence for each dyadic number $0 \leq m \leq 1$ we can define an open set $U(m)$, such that $U(0) \subset A, C U(1) \subset B$ and $U(m) \subset \mathrm{Cl} U\left(m^{\prime}\right)$ for each pair of dyadic numbers $m, m^{\prime}$ such that $0 \leq m<m^{\prime} \leq 1$. Now define, for real number $t \in[0,1]$

$$
U(t)=\bigcup_{m \leq t} U(m)
$$

for $m$ dyadic, hence by the preceding this definition agrees with the preceding one for $t$ dyadic; also if $0 \leq t<t^{\prime} \leq 1$, then there exists two dyadic numbers $m, m^{\prime}$ such that $t \leq m<m^{\prime} \leq t^{\prime}$, and by the preceding we have $U(t) \subset U(m)$, hence $U(t) \subset U(m) \subset \mathrm{Cl} U\left(m^{\prime}\right) \subset \mathrm{Cl} U\left(t^{\prime}\right)$.

The proof is complete.

\section{Extension of a Lower Continuous Real Valued Function}

Let $X$ and $Y$ be two topological spaces and let $A \neq X$ be a closed subset of $X$. If $f$ is a lower continuous mapping of a $A$ into $Y$, it is not always possible extend $f$ to a lower continuous mapping of the whole of $X$ into $Y$. 
When $Y=\mathrm{Cl} \mathbb{R}$, the possibility of such an extension is determined by the following statement.

Theorem 2. A topological space $X$ is lower normal if and only if for given any closed subset $A$ of $X$ and any bijective lower continuous real-value function $f$ (finite or not) defined on $A$, there exists an extension $g$ of $f$ to the whole space $X$, which is a lower continuous mapping of $X$ into $\mathrm{Cl} \mathbb{R}$, i.e., $g \mid A=f$.

Proof. It is easy to see that the equivalent of lower normality in this statement implies the equivalent of lower normality in Theorem 1 ; for if $B$ and $C$ are two disjoint closed subsets of $X$, then the function which is equal to 0 on $B$ and equal to 1 on $C$ has a lower continuous extension $f$ to $X$. If $g=\inf \left\{f^{+}, 1\right\}$, then $g$ is lower continuous on $X$, takes its values in $[0,1]$ and is equal to 0 on $B$ and to 1 on $C$.

Let us show conversely. Since $\mathrm{Cl} \mathbb{R}$ and the interval $[-1,1]$ are homeomorphic, we need consider only the case where the lower continuity mapping $f: A \rightarrow \mathrm{Cl} \mathbb{R}$ takes its values in $[-1,1]$. We shall construct an extension $g$ of $f$ to $X$ by forming a sequence $\left(g_{n}\right)$ of lower continuous functions on $X$, such that the sequence $\left(g_{n}(x)\right)$ lower converges for all $x \in X$ to a point of the interval $[-1,1]$; this limit will, by definition, be the value of $g$ at $x$, and it will follow from the choice of the $g_{n}$ that the function $g$ satisfies the required conditions. The following statement for this is essential.

Proposition 4. Let $X$ be a lower normal space and let $u$ be a lower continuous mapping of closed set $A \subset X$ into $[-1,1]$, then there is a lower continuous mapping $v$ of $X$ into $[-1 / 3,1 / 3]$ such that

$$
|u(x)-v(x)|^{-1} \geq \frac{3}{2} \quad \text { for all } \quad x \in A .
$$

Proof. Let $\mathcal{H}$ be the set of all $x \in A$ such that $-1 \leq u(x) \leq-1 / 3$, and let $\mathcal{K}$ be the set of all $x \in A$ such that $1 / 3 \leq u(x) \leq 1 ; \mathcal{H}$ and $\mathcal{K}$ are closed in $A$, and therefore in $X$, and do not intersect; hence by Theorem 1 there is a lower continuous mapping $v$ of $X$ into $[-1 / 3,1 / 3]$ which is equal $-1 / 3$ on $\mathcal{H}$ and to $1 / 3$ on $\mathcal{K}$. The mapping $v$ satisfies the conditions of the statement.

In the further proof, we now define the functions $g_{n}$ by induction. Applying Proposition 4 with $u=f$, we define $g_{0}$ to be a lower continuous mapping of $X$ into $[-1 / 3,1 / 3]$ such that $\left|f(x)-g_{0}(x)\right|^{-1} \geq 3 / 2$ for all $x \in A$. Suppose now that a lower continuous mapping $g_{n}$ of $X$ into the interval

$$
\left[-1+(2 / 3)^{n+1}, 1-(2 / 3)^{n+1}\right]
$$

has been defined, such that $\left|f(x)-g_{n}(x)\right|^{-1} \geq(3 / 2)^{n+1}$ for all $x \in A$. Applying Proposition 4 to the function $u(x)=(2 / 3)^{n+1}\left(f(x)-g_{n}(x)\right)$, we see that there exists a lower continuous mapping $h_{n+1}$ of $X$ into the interval 
$\left[-2^{n+1} / 3^{n+2}, 2^{n+1} / 3^{n+2}\right]$ such that

$$
\left|f(x)-g_{n}(x)-h_{n+1}(x)\right|^{-1} \geq\left(\frac{3}{2}\right)^{n+2}
$$

for all $x \in A$; the induction is completed by taking $g_{n+1}=g_{n}+h_{n+1}$, since this function satisfies the inequality $\left|g_{n+1}(x)\right| \geq 1-(2 / 3)^{n+2}$ for all $x \in X$, by virtue of the definition of $h_{n+1}$. From this definition it follows that, for $m, n \geq p$, we have

$$
\left|g_{m}(x)-g_{n}(x)\right|^{-1} \geq \frac{3^{p+2}}{2^{p+1}} \sum_{k=0}^{\infty}\left(\frac{3}{2}\right)^{k} \geq\left(\frac{3}{2}\right)^{p+1}
$$

at each point $x \in X$; hence the sequence $\left(g_{n}(x)\right)$ is a lower Cauchy sequence for each $x \in X$, and therefore lower converges to a point $g(x)$ of the interval $[-1,1]$; and since $f(x)-g_{n}(x)$ tends to 0 for all $x \in A$ as $n \rightarrow \infty, g$ is an extension of $f$ to $X$.

It remains therefore only to show that $g$ is lower continuous on $X$. Now let $x$ be any point of $X$; then, given any $\varepsilon>0$, there exists an integer $n_{0}$ such that

$$
\left|g_{m}(y)-g_{n}(y)\right|^{-1} \geq \varepsilon
$$

for all $y \in X$ and all $m \geq n_{0}$ and all $n \geq n_{0}$; hence, letting $m$ tend to $\infty$, we have

$$
\left|g(y)-g_{n}(y)\right|^{-1} \geq \varepsilon .
$$

Let $V$ be a neighborhood of $x$ such that $\left|g_{n}(x)-g_{n}(y)\right|^{-1} \geq \varepsilon$ for all $y \in V$; then, for each $y \in V$ we shall have for $\psi \in \mathcal{D}([0,+\infty])$ that is

$$
\begin{aligned}
& |g(y)-g(x)|^{-1} \\
& \geq \min \left\{\left|g(y)-g_{n}(y)\right|^{-1},\left|g_{n}(y)-g_{n}(x)\right|^{-1},\left|g(x)-g_{n}(x)\right|^{-1},\right. \\
& \left.\psi\left(\left|g(y)-g_{n}(y)\right|^{-1},\left|g_{n}(y)-g_{n}(x)\right|^{-1},\left|g(x)-g_{n}(x)\right|^{-1}\right)\right\} \\
& \geq \min \left\{\left|g(y)-g_{n}(y)\right|^{-1},\left|g_{n}(y)-g_{n}(x)\right|^{-1},\left|g(x)-g_{n}(x)\right|^{-1}\right. \text {, } \\
& \left.\min \left\{\left|g(y)-g_{n}(y)\right|^{-1},\left|g_{n}(y)-g_{n}(x)\right|^{-1},\left|g(x)-g_{n}(x)\right|^{-1}\right\}\right\} \\
& \geq \min \left\{\left|g(y)-g_{n}(y)\right|^{-1},\left|g_{n}(y)-g_{n}(x)\right|^{-1},\left|g(x)-g_{n}(x)\right|^{-1}\right\} \\
& \geq \min \{\varepsilon, \varepsilon, \varepsilon\}=\varepsilon,
\end{aligned}
$$

which shows that $g$ ia a lower continuous at $x$.

The proof is complete. 


\section{Lower Continuous Partitions of Unity}

Let $X$ be topological space and let $f$ be a real valued function defined on $X$. The support of $f$, denoted by $\operatorname{Supp}(f)$ is the smallest closed set $S$ in $X$ such that $f(x)=0$ for all $x \notin S$.

Let $\left(f_{i}\right)_{i \in I}$ be a family of finite real valued functions on $X$ whose supports form a locally finite family, where $I$ is a well order the index set; then the sum $\sum_{i \in I} f_{i}(x)$ is defined for each $x \in X$ (since it contains only a finite number of non-zero terms). The finite real valued function $x \mapsto \sum_{i \in I} f_{i}(x)$ is called the sum of the family $\left(f_{i}\right)_{i \in I}$, and is denoted by $\sum_{i \in I} f_{i}$. If each of the $f_{i}$ is lower continuous, then so is $f=\sum_{i \in I} f_{i}$; for if $x$ is any point of $X$, there is a neighborhood $V$ of $x$ which meets only a finite number of supports of the $f_{i}$, and hence there is a finite subset $\mathcal{H}$ of $I$ such that $f(y)=\sum_{i \in \mathcal{H}} f_{i}(y)$ for all $y \in V$.

Theorem 3. Let $\left(A_{i}\right)_{i \in I}$ be a locally finite open covering of a closed set $Y$ in a lower normal space $X$. Then there is an open covering $\left(B_{i}\right)_{i \in I}$ of $Y$ such that $C A_{i} \subset \mathrm{Cl} B_{i}$ for each $i \in I$.

Proof. We shall define a family $\left(B_{i}\right)_{i \in I}$ of open sets in $X$, by transfinite induction, such that:

(a) $A_{i} \subset \mathrm{Cl} B_{i}$ for each $i \in I$, and

(b) for each $i \in I$, the family formed by the $B_{\alpha}$ such that $\alpha \leq i$ and by the $A_{\alpha}$ such that $\alpha>i$ is an open covering of $Y$.

Suppose that we have defined the $B_{i}$ for $i<\beta$, so that (a) and (b) are satisfied for all $i<\beta$, and let us show that we can define $B_{\beta}$ in such a way that (a) and (b) are also satisfied for $i=\beta$.

Let us first show that the $B_{i}$ for which $i<\beta$ and the $A_{i}$ for which $i \geq \beta$ form a covering of $Y$. By hypothesis, for each $x \in Y$ there is only a finite number of indices $\alpha \in I$ such that $x \in A_{\alpha}$, say $\alpha_{1}<\alpha_{2}<\ldots<\alpha_{n}$; let $\alpha_{k}$ be the greatest of the $\alpha_{i}$ such that $\alpha_{i}<\beta$; if $k<n$ we have $x \in A_{\alpha(n)}$ and $\alpha(n) \geq \beta$, and if $k=n$ the inductive hypothesis shows that $x$ belongs to some $B_{\alpha}$ such that $\alpha \leq \alpha_{n} \leq \beta$, and our assertion follows. Now put $G=(C Y) \cup\left(\cup_{i<\beta} B_{i}\right) \cup\left(\cup_{i>\beta} A_{i}\right) ; G$ is open, and from what has been said we have $C A_{\beta} \subset G$; by virtue of Proposition 3 for lower normal spaces, there is therefore an open set $V$ such that $C A_{\beta} \subset G \subset \mathrm{Cl} V$. If we put $B_{\beta}=V$, we have $C A_{\beta} \subset G \subset \mathrm{Cl} B_{\beta}$ and $B_{\beta} \cup G=X$, so that the $B_{i}$ such that $i \leq \beta$ and the $A_{i}$ such that $i>\beta$ cover $Y$.

The proof is complete.

A second proof of this statement we can give via the Axiom of Choice. For the normal spaces a suitable statement appeared in Lefschetz [4].

On the other hand, if $X$ is a topological space and all sets $A_{i}(i \in I)$ are open (closed), we say that the cover $\left\{A_{i}\right\}_{i \in I}$ is open (closed). A family $\left\{A_{i}\right\}_{i \in I}$ of subsets of a set $X$ is called point-finite (point-countable) if for 
every $x \in X$ the set $\left\{i \in I: x \in A_{i}\right\}$ is finite (countable). Clearly every locally finite cover is point finite.

In this sense, via Zorn's lemma, we notice that the following general statement holds: For every point-finite open cover $\left\{U_{i}\right\}_{i \in I}$ of a lower normal space $X$ there exists an open cover $\left\{V_{i}\right\}_{i \in I}$ of $X$ such that $C U_{i} \subset \mathrm{Cl}\left(V_{i}\right)$ for every $i \in I$.

In connection with the preceding, given a family $\left(A_{i}\right)_{i \in I}$ of subsets of a topological space $X$, a family $\left(f_{i}\right)_{i \in I}$ of real-valued functions defined on $X$ is said to be subordinate to the family $\left(A_{i}\right)_{i \in I}$ if we have $\operatorname{Supp}\left(f_{i}\right) \subset A_{i}$ for each index $i \in I$.

In this sense, a lower continuous partition of unity on $X$ is any family $\left(f_{i}\right)_{i \in I}$ of nonnegative real valued lower continuous functions on $X$ whose supports form a locally finite family and which are such that $\sum_{i \in I} f_{i}(x)=1$ for all $x \in X$.

Proposition 5. Given any locally finite open covering $\left(A_{i}\right)_{i \in I}$ of a lower normal space $X$, there exists a lower continuous partition of unity $\left(f_{i}\right)_{i \in I}$ on $X$, subordinate to the covering $\left(A_{i}\right)_{i \in I}$.

Proof. By Theorem 3 there exists an open covering $\left(B_{i}\right)_{i \in I}$ of $X$ such that $C A_{i} \subset \mathrm{Cl} B_{i}$ for each $i \in I$, and it is clear that the covering $\left(B_{i}\right)$ is locally finite. By Proposition 3, for each $i \in I$ there exists an open set $W_{i}$ such that $A_{i} \subset W_{i} \subset \mathrm{Cl} W_{i} \subset C B_{i}$. By Theorem 1, for each $i \in I$ there exists a lower continuous mapping $g_{i}$ of $X$ into [0,1], such that $g_{i}(x)=1$ on $A_{i}$ and such that the support of $\widetilde{g}_{i}$ is contained in $\mathrm{Cl} W_{i}$, and therefore contained in $B_{i}$. Since $\left(B_{i}\right)$ is a covering of $X$, we have $\sum_{i \in I} g_{i}(x)>0$ for each $x \in X$; if we put

$$
f_{i}(x)=\frac{g_{i}(x)}{\sum_{i \in I} g_{i}(x)} \quad \text { for all } \quad x \in X,
$$

and for all $i \in I$, then the $f_{i}$ form a lower continuous partition of unity subordinate to the covering $\left(B_{i}\right)_{i \in I}$.

The proof is complete.

Corollary 2. Given any locally finite open covering $\left(A_{i}\right)_{i \in I}$ of a closed set $F$ in a lower normal space $X$, there exists a family $\left(f_{i}\right)_{i \in I}$ of nonnegative lower continuous real valued functions on $X$, subordinate to the covering $\left(A_{i}\right)_{i \in I}$ and such that $\sum_{i \in I} f_{i}(x)=1$ for all $x \in F$ and $\sum_{i \in I} f_{i}(x) \leq 1$ for all $x \in X$.

Proof. The family of sets consisting of $C F$ and the $A_{i}$ is a locally finite open covering of $X$. There is therefore a lower continuous partition of unity subordinate to this covering, consisting of a family $\left(f_{i}\right)_{i \in I}$ such that $\operatorname{Supp}\left(f_{i}\right) \subset A_{i}$ for each $i \in I$, and a function $g$ whose support is contained in the complement of $F$. The family $\left(f_{i}\right)_{i \in I}$ clearly satisfies the required conditions.

The proof is complete. 
Proposition 6. Let $(X, \rho)$ be a lower transversal space, where $x \mapsto \rho(x, S)$ is a lower continuous mapping for a set $S \subset X$. If $A$ and $B$ are nonempty closed disjoint subsets of $X$, then there is a lower continuous mapping $g$ : $X \mapsto[0,1] \subset \mathbb{R}$ such that $g \mid A=0$ and $g \mid B=1$.

Proof. Since $A$ (as and $B$ ) a closed set in $X$, thus we have $\rho(x, A)=+\infty$ (as and $\rho(x, B)=+\infty$ ) if and only if $x \in A$ (i.e., $x \in B$ ). Also $\rho(x, A)+$ $\rho(x, B)>0$ for every $x \in X$ because $A$ and $B$ are disjoint subsets. Then the form

$$
g(x)=\frac{\rho(x, A)}{\rho(x, A)+\rho(x, B)} \quad \text { for } \quad x \in X
$$

is a lower continuous function of $X$ into $[0,1]$ such that $g(x)=0$ for $x \in A$ and $g(x)=1$ for $x \in B$.

The proof is complete.

Let $X$ be a linear space over $\mathbb{K}$. The mapping $x \mapsto\|x\|: X \rightarrow[0,+\infty]$ is called a lower transversal seminorm (or lower seminorm) iff: $\|x\| \geq 0$ for every $x \in X,\|\lambda x\|=|f(\lambda)|\|x\|$ for all $\lambda \in \mathbb{K}$ and $x \in X$, where given some $f: \mathbb{K} \rightarrow \mathbb{K}$, and if there is a function $d:[0,+\infty]^{2} \rightarrow[0,+\infty]$ such that

$$
\|x+y\| \geq \min \{\|x\|,\|y\|, d(\|x\|,\|y\|)\}
$$

for all $x, y \in X$.

Further, $x \mapsto\|x\|$ is called a lower transversal norm (or lower norm) iff in addition: $\|x\|=+\infty$ if and only if $x=0$.

A lower transversal normed space $(X,\|\cdot\|)$ over $\mathbb{K}$ consists of a linear space $X$ over $\mathbb{K}$ together with a lower transversal norm $x \mapsto\|x\|$.

The function $d:[0,+\infty]^{2} \rightarrow[0,+\infty]$ in (Nl) is called lower bisection function. From $(\mathrm{Nl})$ it follows, by induction, that there is a function $G$ : $[0,+\infty]^{n} \rightarrow[0,+\infty]$ such that

$$
\left\|x_{0}-x_{n}\right\| \geq
$$

$$
\geq \min \left\{\left\|x_{0}-x_{1}\right\|, \ldots,\left\|x_{n-1}-x_{n}\right\|, G\left(\left\|x_{0}-x_{1}\right\|, \ldots,\left\|x_{n-1}-x_{n}\right\|\right)\right\}
$$

for all $x_{0}, x_{1}, \ldots, x_{n} \in X$ and for any fixed integer $n \geq 2$.

It is easy to verify that the lower transversal normed linear space $X$ is a transversal lower space (see: Tasković [7]) with respect to the lower transverse $\rho: X \times X \rightarrow[0,+\infty]$ defined by

$$
\rho[x, y]=\|x-y\| \quad \text { for all } \quad x, y \in X
$$

thus we obtain $\rho[x-z, y-z]=\rho[x, y]$ and $\rho[\lambda x, \lambda y]=|f(\lambda)| \rho[x, y]$ for all $x, y, z \in X$ and for every scalar $\lambda \in \mathbb{K}$.

In this sense, the sequence $\left\{x_{n}\right\}_{n \in \mathbb{N}}$ in $(X,\|\|$.$) converges (or lower con-$ verges) to $x \in X$ if the sequence $\left\{x_{n}\right\}_{n \in \mathbb{N}}$ converges (or lower converges) in $(X, \rho)$, i.e., if

$$
\rho\left[x_{n}, x\right]=\left\|x_{n}-x\right\| \rightarrow+\infty \quad \text { as } \quad n \rightarrow \infty .
$$


In this sense, a lower transversal normed space $X$ is said to be lower complete (or complete) if it is lower complete as a transversal lower space.

We will, in further, denote by $\mathcal{D}([0,+\infty])$ the set of all lower bisection functions $d:[0,+\infty]^{2} \rightarrow[0,+\infty]$ which are increasing satisfying $d(t, t) \geq t$ for every $t \in[0,+\infty]$.

A function $f: D \rightarrow \mathbb{R}$, where $\mathbb{R}$ denotes the real line in $D$ is a convex subset of $\mathbb{R}^{n}$, is said to be general concave iff there is a function $d$ : $(f(D))^{2} \rightarrow \mathbb{R}$ such that

$$
f(\lambda x+(1-\lambda) y) \geq \min \{f(x), f(y), d(f(x), f(y))\}
$$

for all $x, y \in D$ and for arbitrary $\lambda \in[0,1]$. For this see: Tasković [6].

We notice that lower transversal norm $x \mapsto\|x\|$ is a general concave function. The proof is simple.

Proposition 7. Let $(X, \rho)$ be a lower transversal space with a bisection function $d \in \mathcal{D}([0,+\infty])$ and let $T: M \subset X \rightarrow Y$ be a lower continuous operator on a nonempty closed subset $M$ of $X$ to the lower normed space $Y$. Then $T$ has a lower continuous extension $\widetilde{T}: X \rightarrow \operatorname{conv}(T(M))$, where conv denotes convex hull.

We notice that in the special case $X=\mathbb{R}$ and $M=[a, b]$ for $a, b \in \mathbb{R}$ this statement says that every lower continuous real function $T:[a, b] \rightarrow[\alpha, \beta]$ for $\alpha, \beta \in \mathbb{R}$ can be extended to a lower continuous function $\widetilde{T}: \mathbb{R} \rightarrow[\alpha, \beta]$.

Proof of Proposition 7. To each $y \in X \backslash M$ we assign an open ball $U_{y}$ with $\operatorname{diam}\left(U_{y}\right)>\rho\left(U_{y}, M\right)$. This gives us a covering $\left(U_{y}\right)$ of $X \backslash M$. For this covering, from Proposition 5, there is a partition of unity,

$$
\sum_{j \in J} f_{j}(x)=1 \quad \text { for all } \quad x \in X \backslash M,
$$

where $f_{j}: X \backslash M \rightarrow[0,1]$ is lower continuous for all $j \in J$ and zero outside of $U_{y(j)}$ for suitable $y(j)$, while each $x \in X \backslash M$ has a neighborhood $V(x)$ such that all but a finite number of $f_{j}$ are identically zero on $V(x)$.

For each $y \in X \backslash M$ we choose an $m_{y} \in M$ such that $\rho\left(m_{y}, U_{y}\right)>$ $2 \rho\left(M, U_{y}\right)$ and define

$$
\widetilde{T}(x)= \begin{cases}T(x) & \text { if } x \in M, \\ \sum_{j \in J} f_{j}(x) T\left(m_{y(j)}\right) & \text { if } x \in X \backslash M ;\end{cases}
$$

it follows: from $(2)$ that $\widetilde{T}(x) \subset \operatorname{conv}(T(M))$, and that $\widetilde{T}$ is clearly lower continuous on $\operatorname{Int}(M)$ and $X \backslash M$. We show that $\widetilde{T}$ is lower continuous on $\partial M$. In this sense let $x_{0} \in \partial M$. Then, $\widetilde{T}\left(x_{0}\right)=T\left(x_{0}\right)$. If $x \in X \backslash M$ and $f_{j}(x) \neq 0$, then by the construction of $f_{j}$, we have $x \in U_{y}$, where we set 
$y=y(j)$. Applying the inequality (Am), since $d \in \mathcal{D}([0,+\infty])$, yields

$$
\begin{aligned}
\rho\left(m_{y}, x\right) & \geq \min \left\{\rho\left(m_{y}, U_{y}\right), \operatorname{diam}\left(U_{y}\right), d\left(\rho\left(m_{y}, U_{y}\right), \operatorname{diam}\left(U_{y}\right)\right)\right\} \geq \cdots \\
\cdots & \geq \min \left\{\rho\left(m_{y}, U_{y}\right), \operatorname{diam}\left(U_{y}\right), \min \left\{\rho\left(m_{y}, U_{y}\right), \operatorname{diam}\left(U_{y}\right)\right\}\right\} \geq \\
& \geq \min \left\{\rho\left(M, U_{y}\right), 2 \rho\left(M, U_{y}\right)\right\}=2 \rho\left(M, U_{y}\right),
\end{aligned}
$$

and therefore

$$
\begin{aligned}
\rho\left(m_{y}, x_{0}\right) & \geq \min \left\{\rho\left(m_{y}, x\right), \rho\left(x, x_{0}\right), d\left(\rho\left(m_{y}, x\right), \rho\left(x, x_{0}\right)\right)\right\} \geq \cdots \\
\cdots & \geq \min \left\{\rho\left(m_{y}, x\right), \rho\left(x, x_{0}\right), \min \left\{\rho\left(m_{y}, x\right), \rho\left(x, x_{0}\right)\right\}\right\} \geq 2 \rho\left(x, x_{0}\right) .
\end{aligned}
$$

Since $0 \leq f_{j}(x) \leq 1$ and $f_{j}(x)=0$ for $\rho\left(m_{y(j)}, x_{0}\right)<2 \rho\left(x, x_{0}\right)$ we have the following inequality in the form

$$
\left\|\widetilde{T}(x)-\widetilde{T}\left(x_{0}\right)\right\|=\left\|\sum_{j \in J} f_{j}(x)\left(T\left(m_{y(j)}\right)-T\left(x_{0}\right)\right)\right\| \geq \inf R(x)
$$

for all $x \in X \backslash M$, where we define the set $R(x)$ in the form

$$
R(x)=\left\{\left\|T\left(m_{y(j)}\right)-T\left(x_{0}\right)\right\|: j \in J, \rho\left(m_{y(j)}, x_{0}\right) \geq 2 \rho\left(x, x_{0}\right)\right\} .
$$

If $x_{n} \in X \backslash M$ for all $n \in \mathbf{N}$ and $x_{n} \rightarrow x_{0}$ as $n \rightarrow \infty$, then the lower continuity of $T$ implies that $\inf R\left(x_{n}\right) \rightarrow+\infty$ as $n \rightarrow \infty$. Hence, $\widetilde{T}\left(x_{n}\right) \rightarrow \widetilde{T}\left(x_{0}\right)$ as $n \rightarrow \infty$. In the alternative, if $x_{n} \in M$ for all $n \in \mathbf{N}$ and $x_{n} \rightarrow x_{0}$ as $n \rightarrow \infty$, then $T\left(x_{n}\right)=\widetilde{T}\left(x_{n}\right)$, so that $\widetilde{T}\left(x_{n}\right) \rightarrow \widetilde{T}\left(x_{0}\right)$ as $n \rightarrow \infty$. Thus, $\widetilde{T}$ is lower continuous at $x_{0} \in \partial M$.

The proof is complete.

\section{REFERENCES}

[1] P. Alexandroff and P. Urysohn, Zur Theorie der topologischen Räume, Math. Annalen, 92 (1924), 258-266.

[2] J. Dieudonné, Une généralisation des espaces compacts, Journ. de Math. 23 (1944), $65-76$.

[3] H. Lebesgue: Sur le problème des Dirichlet, Rend. Circ. mat. di Palermo, 24 (1907), 371-402.

[4] S. Lefschetz: Algebraic topology, Amer. Math. Soc. New York, 1942.

[5] M.R. Tasković: Transversal spaces, Math. Moravica, 2 (1998), 133-142.

[6] M.R. Tasković, Nonlinear Functional Analysis, Second Book: Monographs - Global Convex Analysis - General convexity, Variational methods and Optimization, Zavod za udžbenike i nastavna sredstva i Vojnoizdavački zavod, Beograd 2001, (in Serbian), 1223 pages.

[7] M.R. Tasković: Survey on transversal normed spaces, Math. Moravica, 7 (2003), $149-170$. 
[8] H. Tietze: Über Funktionen, die auf einer abgeschlossen Menge stetig sind, J. Reine Angew. Math. 145 (1915), 9-14.

[9] A. Tychonoff: Über einen Metrisationssatz von P. Urysohn, Math. Annalen, 95(1925), 139-142.

[10] P. Urysohn: Über die Mächtigkeit der zusammenhängenden Mengen, Math. Annalen, 94 (1925), 262-295.

[11] L. Vietoris, Stetige Mengen, Monatsh. für Math. und Phys., 31 (1921), 173-204.

\section{Milan R. TAsković}

FaCUlty of Mathematics

P.O. Box 550

11000 BEOGRAD

SERBia

Home address:

Milan R. Tasković

NeHruOva 236

11070 BELGRADE

SERBIA

E-mail address: andreja@predrag.us 